Artigo Original

\title{
Avaliação da qualidade de vida, da dor nas costas, da funcionalidade e de alterações da coluna vertebral de estudantes de fisioterapia
}

\author{
Caroline de Andrade Bacchi ${ }^{1}$ \\ Cláudia Tarragô Candotti ${ }^{2}$ \\ Matias Noll ${ }^{3}$ \\ Carlos Eduardo dos Santos Minossi ${ }^{1}$ \\ ${ }^{1}$ Universidade Federal do Rio Grande do Sul, Porto Alegre, RS, Brasil \\ ${ }^{2}$ Curso de Fisioterapia e Educação Física e do Programa de Pós-Graduação em Ciências do \\ Movimento Humano da Universidade Federal do Rio Grande do Sul, Porto Alegre, RS, Brasil \\ ${ }^{3}$ Escola Superior de Educação Física, Universidade Federal do Rio Grande do Sul, Porto Alegre, \\ RS, Brasil

\begin{abstract}
Resumo: O objetivo deste estudo foi avaliar a qualidade de vida, dor nas costas, funcionalidade e as alterações da coluna vertebral de estudantes de fisioterapia. Participaram 42 universitários, sendo avaliada a coluna vertebral por meio do arcômetro, a dor nas costas por meio de um questionário multidimensional de dor, a qualidade de vida por meio do questionário SF-36 e funcionalidade a partir do Roland-Morris. Foi realizada estatística descritiva e teste qui-quadrado $(\alpha=0,05)$. Os resultados demonstraram: prevalência de alterações nas curvaturas da coluna vertebral de 38,1\%; $(n=16)$; alta prevalência de dor nas costas (69\%; $n=29)$; baixa prevalência de comprometimento da funcionalidade $(3,4 \% ; n=1)$; que não há associação entre as alterações nas curvaturas da coluna vertebral e funcionalidade e dor nas costas; e que os escores dos domínios do SF-36 foram maiores que 45, exceto o domínio "dor", cujo escore aproximado foi de 35. Conclui-se que quanto menor os níveis de intensidade da dor melhor o nível de qualidade de vida.
\end{abstract}

Palavras-chave: Coluna vertebral. Dor. Qualidade de vida.

\section{Assessment of quality of life, back pain, functionality and changes of the spine of physical therapy students}

\begin{abstract}
The objective was to evaluate the quality of life, back pain, function and the changes of the spine of physical therapy students. 42 students participated in this study. We assessed the spine through the arcometer, back pain using a multidimensional pain questionnaire, quality of life using the SF-36 and feature from the Roland-Morris. Descriptive statistics and chi-square test $(\alpha=0.05)$ was performed. The results demonstrated a prevalence of changes in curvatures of the spine $38,1 \%(n=16)$, high prevalence of back pain $(69 \%, n=29)$, low prevalence of impaired functionality $(3.4 \%, n=1)$, there is no association between changes in the curvatures of the spine and back pain and functionality, and SF-36 domain scores were higher than 45, except in "pain" area (score $=35$ ). Conclusion: the lower the levels of pain intensity level, best the quality of life.
\end{abstract}

Keywords: Spine. Pain. Quality of life.

\section{Introdução}

Atualmente observa-se um incremento do número de pessoas que sofrem com problemas na coluna vertebral, como a ocorrência de dor e de alterações posturais (KENDALL et al., 2007). Tem sido relatado que estes problemas estão associados com baixa auto-estima, redução da qualidade de vida, limitação funcional (HINMAN, 2004, DIEEN et al., 2003) e, principalmente, relacionados a fatores estressores e externos na vida diária dos indivíduos, especialmente aqueles cujas atividades cotidianas exigem um longo período em posição sentada (CARNEIRO et al., 2005; CANDOTTI, NOLL e CRUZ, 2010).
Para realizar a avaliação das alterações posturais da coluna vertebral tradicionalmente são utilizados métodos de avaliação indiretos e diretos (D'OSUALDO et al., 1997). O método indireto mais utilizado entre os fisioterapeutas é a avaliação postural, que corresponde a uma análise visual qualitativa, dependente da experiência palpatória do avaliador e que não permite a quantificação em graus das alterações da coluna vertebral (TRIBASTONE, 2000; FALCÃO et al., 2007), tornando-se, então, um método de avaliação subjetivo e limitado. Portanto, muitas vezes há necessidade da utilização de métodos de avaliação diretos que 
permitam análises quantitativas e objetivas, seja por técnicas invasivas ou não-invasivas.

O método padrão-ouro utilizado para a avaliação das curvaturas fisiológicas da coluna vertebral e de patologias da coluna é o raio-X. No entanto, muitos autores enfatizam as desvantagens desse método: exposição do paciente à radiação ionizante, má qualidade da imagem que pode dificultar a análise do exame e ausência do equipamento no ambiente clínico dos fisioterapeutas para acompanhamento evolutivo dos pacientes (BARAÚNA et al., 2005; DOODY et al., 2000). Assim, uma alternativa considerada eficaz na avaliação das curvaturas da coluna é a utilização das chamadas técnicas não-invasivas, as quais, a partir de instrumentalização de baixa complexidade permitem avaliar de forma quantitativa as curvaturas da coluna vertebral (LEROUX, 2001; BARAÚNA et al., 2005; CASTRO e LOPES, 2003). Dentre os diversos instrumentos referidos na literatura, destacam-se 0 arcômetro (D'OSUALDO et al., 1997; CHAISE et al., 2011) e o flexicurva (HINMANN, 2004), por serem considerados de fácil manuseio, baixo custo e por permitirem a mensuração quantitativa em graus das curvaturas da coluna vertebral.

Empiricamente, é aceito que as dores referidas na região das costas podem ter ligação com possíveis alterações das curvaturas fisiológicas da coluna vertebral, muitas vezes provenientes de maus hábitos posturais nas atividades da vida diária, como por exemplo, a permanência por longo período em uma posição incorreta, fatores ambientais inadequados, sejam familiares, profissionais ou de estudo, nível de atividade física, estilo de vida sedentário, utilização de sapatos com saltos altos ou ainda possíveis patologias ortopédicas (KNOPLICH , 2003; CAILLIET, 2001; CANDOTTI, NOLL e CRUZ, 2010).

Considerando a existência de dor nas costas como um fator limitante da aptidão física, emocional e cognitiva de um indivíduo, e em especial de estudantes universitários, capaz de limitar e/ou modificar aspectos da qualidade de vida e da capacidade funcional, entende-se que é de fundamental importância a realização de avaliação postural para investigar a presença de alterações na coluna vertebral assim como identificar o quanto a dor nas costas dos estudantes está relacionada com estas alterações. Ainda, a investigação da capacidade funcional e da percepção da qualidade de vida (TSUKIMOTO et al., 2006; OCARINO et al., 2009) dos estudantes poderão fornecer uma ampla possibilidade de compreensão dos problemas na coluna vertebral desta população. Deste modo, justifica-se o presente estudo, o qual teve como objetivo avaliar a qualidade de vida, a dor nas costas, a funcionalidade e as alterações da coluna vertebral de estudantes de fisioterapia.

\section{Tipo de Estudo}

\section{Método}

$O$ delineamento desta pesquisa é do tipo Estudo de Caso Institucional (GAYA, 2008), sendo utilizadas como estratégias para apresentação dos resultados abordagens descritivas e inferenciais das variáveis do estudo.

\section{Amostra}

A amostra foi constituída por 42 estudantes voluntários matriculados no curso de Bacharelado em Fisioterapia da Universidade Federal do Rio Grande do Sul no ano de 2010. Os critérios de exclusão foram: 1) impossibilidade do indivíduo de permanecer na postura ortostática, 2) existência de qualquer intervenção cirúrgica prévia na coluna vertebral e 3) gravidez.

Para caracterizar a amostra foram mensuradas a massa corporal, a estatura e os perímetros da cintura e do quadril, utilizando uma balança digital (Filizola), um estadiômetro (Filizola) e uma fita métrica (Sanny), respectivamente. Foram também calculados 0 Índice de Massa Corporal (IMC) e a Relação Cintura Quadril (RCQ), obtida pelo quociente entre o perímetro da cintura $(\mathrm{cm})$ e o perímetro do quadril $(\mathrm{cm})$. Este estudo foi aprovado no Comitê de Ética e Pesquisa da UFRGS, sob número 19191.

Para este estudo foi utilizado o instrumento arcômetro e questionários para avaliação da dor nas costas, da qualidade de vida e da funcionalidade.

\section{Instrumento avaliativo da coluna vertebral: Arcômetro}

O arcômetro (D'OSUALDO et al., 1997; DIAS et al., 2008; CHAISE et al., 2011) é um instrumento constituído por: (1) uma haste principal de $50 \mathrm{~cm}$ de comprimento, $1 \mathrm{~cm}$ de largura e $1 \mathrm{~cm}$ de espessura; (2) duas hastes menores, perpendiculares a principal, com comprimento de $40 \mathrm{~cm}$, largura e espessura de 
$0,5 \mathrm{~cm}$ de cada; (3) uma haste central, também perpendicular a principal, com comprimento de 40 $\mathrm{cm}$, largura e espessura de $0,5 \mathrm{~cm}$ cada; (4) três conexões primárias, que permitem a movimentação das duas hastes menores e da haste central na vertical; e uma conexão secundária, permitindo a movimentação destas hastes também na horizontal (Figura 1). Cada conexão primária é constituída por duas peças, sendo uma no formato de "U" e uma peça reta para fixação da haste central. As conexões secundárias são constituídas somente de uma peça em formato de "U". Nas hastes foram acopladas escalas métricas, permitindo mensurações lineares nas direções vertical e horizontal. Na haste principal, em uma das faces de $0,25 \mathrm{~cm}$ foi fixada e centralizada uma escala em milímetros, de 0 a $80 \mathrm{~cm}$, partindo do ponto de fixação inicial da primeira haste fixada. Na haste central, também foi fixada e centralizada uma escala milimétrica, de 0 a $50 \mathrm{~cm}$ (Figura 1).

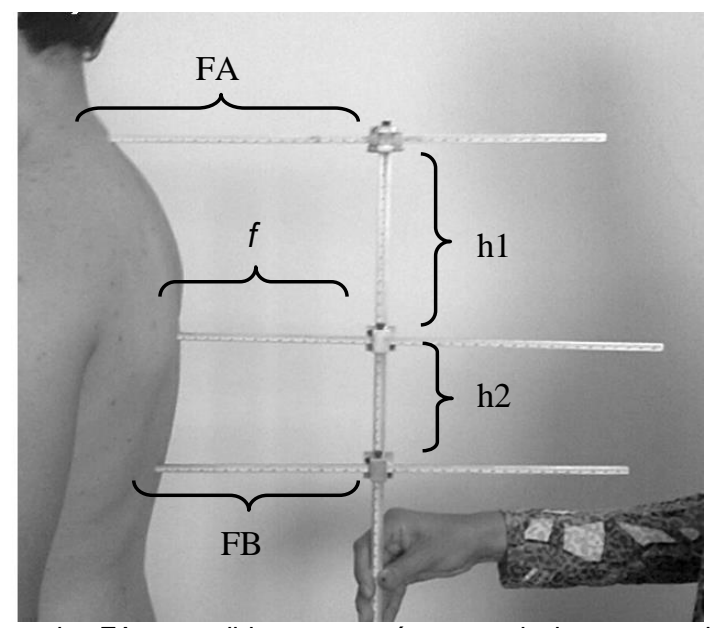

Legenda: $\mathrm{FA}=$ medida, em centímetros, da haste superior; $\mathrm{FB}=$ medida, em centímetros, da haste inferior; $f=$ medida, em centímetros, da haste central colocada no ápice da curvatura; $\mathrm{h} 1=$ medida, em centímetros, que corresponde à reta gerada entre a vértebra limite superior e o ponto do ápice da curvatura; h2= medida, em centímetros, que corresponde à reta gerada entre a vértebra limite inferior e o ponto do ápice da curvatura.

Figura 1. Instrumento arcômetro posicionado com respectivas ilustrações das variáveis de medida.

\section{Instrumento avaliativo da dor nas costas}

Para avaliação da dor nas costas foi utilizado um questionário de dor proposto por Candotti e Guimarães (1998), cujo objetivo foi coletar informações sobre a existência e intensidade da dor. Para análise da existência de dor foram consideradas duas categorias: com dor e sem dor. Para a análise da intensidade da dor, a escala de 0 a 10 do questionário foi dividida em 4 categorias, segundo a seguinte classificação: de 1 à 3 , dor fraca; de 4 à 6 , dor moderada; de 7 à 9 , dor forte; e 10, dor muito forte (CIENA et al., 2008).

\section{Instrumento avaliativo da qualidade de vida}

Para avaliação da qualidade de vida foi utilizado o questionário SF-36. Este questionário foi traduzido e validado para a língua portuguesa por Ciconelli et al em 1997, sendo constituído por 36 itens divididos em 8 domínios: capacidade funcional, aspectos físicos, dor, estado geral de saúde, vitalidade, aspectos sociais, aspectos emocionais e saúde mental. Este questionário apresenta um escore final de 0 a 100 , no qual o 0 corresponde ao pior estado geral de saúde e 100 ao melhor estado de saúde (CICONELLI et al., 1997). Cada dimensão do questionário é avaliada em separado, visto que não existe um único valor que possa sintetizar a avaliação da qualidade de vida (TSUKIMOTO et al., 2006).

\section{Instrumento avaliativo da funcionalidade}

O questionário Roland-Morris foi utilizado para avaliar o desempenho funcional e foi preenchido apenas pelos estudantes que referiram dor nas costas (VIGATTO et al., 2007). A validação deste instrumento para a língua portuguesa foi realizada por Nusbaum et al (2001). O escore do questionário Roland-Morris é de zero à 24 pontos, e o ponto de corte corresponde ao escore igual ou superior à 14, caracterizando a presença de incapacidade significante decorrente da dor na região do tronco (coluna torácica e lombar) (TSUKIMOTO et al., 2006, OCARINO et al., 2009). Portanto, para análise da variável funcionalidade foram consideradas duas categorias: com limitação e sem limitação.

\section{Procedimentos}

Os procedimentos para avaliar a presença de alterações nas curvaturas da coluna vertebral no plano sagital, a dor nas costas e a qualidade de vida nos estudantes de fisioterapia foram realizados nessa ordem sequencial, no mesmo dia e local, pela mesma equipe avaliadora. Primeiramente, os estudantes foram convidados a participar do estudo, sendo realizada uma breve explicação sobre o mesmo e apresentado do TCLE. Logo após os estudantes responderam o questionário sobre a qualidade de vida e da dor nas costas. Os estudantes que referiram sentir dor nas costas preencheram também o questionário sobre funcionalidade.

Após o preenchimento dos questionários foram avaliadas as curvaturas da coluna vertebral 
dos estudantes. Os estudantes foram posicionados em postura ortostática, braços relaxados ao longo do corpo, pés paralelos sem calçados e com o dorso despido. O avaliador, através do método palpatório, identificou e demarcou os processos espinhosos das vértebras torácicas (T1, T12) e lombares (L1 e L5) do estudante (Figura 2a). Para mensurar as curvaturas torácica e lombar com o arcômetro, o avaliador posicionou as hastes do instrumento sobre os processos espinhosos demarcados. Nesta etapa obteve-se as medidas de FA e FB (Figura 1). Logo após, o avaliador identificou 0 ápice da curvatura através da haste central que corresponde à medida de f. Em seguida, realizou a leitura na escala do instrumento das medidas de h1 e h2 que correspondem à distância entre a vértebra limite superior e o ponto do ápice da curvatura, e entre, o ápice da curvatura e a vértebra limite inferior, respectivamente. A verticalidade do arcômetro durante a coleta foi garantida pelo marcador de nível acoplado na haste superior. Esse procedimento foi repetido tanto para a obtenção dos dados da curvatura torácica (Figura 2b) quanto para a curvatura lombar (Figura 2c).
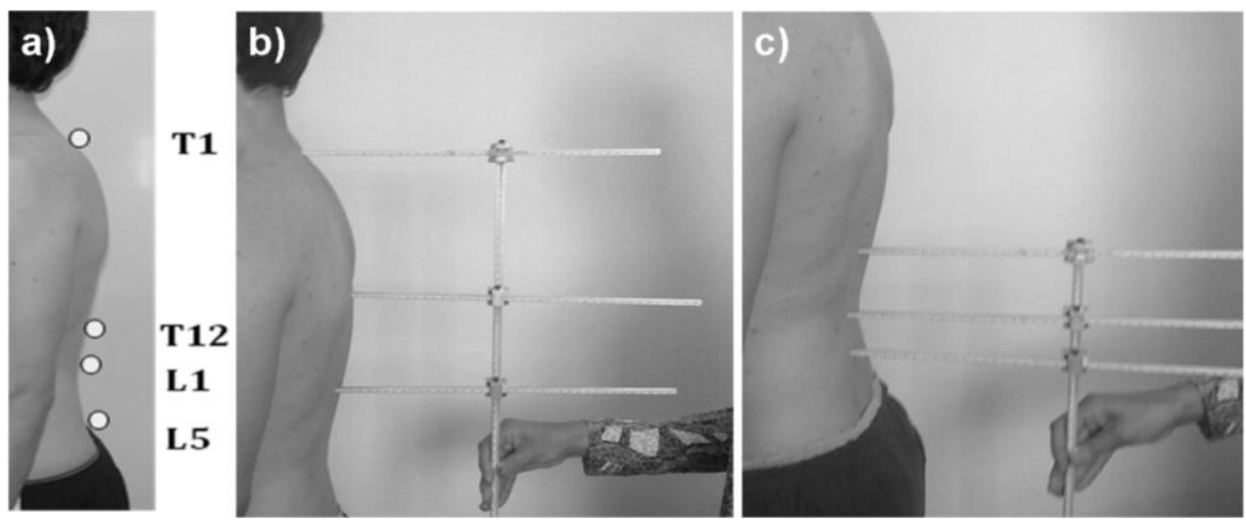

Figura 2. Avaliação da coluna vertebral: (a) marcação dos pontos anatômicos e mensuração com o arcômetro (b) da região torácica e (c) da região lombar.

Os dados provenientes do arcômetro, ou seja, valores de h1, h2, f, FA e FB (Figura 1) da curvatura torácica e lombar dos indivíduos foram inseridos nas equações 1 à 5 , de modo que a partir de três relações trigonométricas foram calculados os graus das curvaturas torácica e lombar (HAISE et al., 2011).

$$
\begin{aligned}
& f 1=F A-f \\
& f 2=F B-f \\
& \varphi 1=180^{\circ}-2^{\circ} \operatorname{atan}\left(\frac{\mathrm{f} 1}{\mathrm{~h} 1}\right) \\
& \varphi 2=180^{\circ}-2^{\circ} \operatorname{atan}\left(\frac{\mathrm{f} 2}{\mathrm{~h} 2}\right) \\
& \varphi 1+\varphi 2=A A
\end{aligned}
$$

onde,

$\varphi 1$ ângulo, em graus, correspondente ao primeiro arco gerado a partir dos valores de f1 e h1; $\varphi 2=$ ângulo, em graus, correspondente ao segundo arco gerado a partir dos valores de f2 e h2; AA = ângulo do arcômetro em graus.

Para classificação da coluna torácica foram utilizados os parâmetros propostos por Bernhartd e Bridwell (1989), onde a postura é considerada normal quando os valores angulares da curvatura torácica variam entre $20^{\circ}$ e $60^{\circ}$. Quando o valor da curvatura apresentou-se maior que $60^{\circ}$ foi classificada como hipercifose torácica e quando o valor da curvatura foi menor que $20^{\circ}$ foi classificada como retificação torácica. Para a classificação da coluna lombar os parâmetros de referência normais variam entre $22^{\circ}$ e $54^{\circ}$ (PROPST-PROCTOR e BLECK, 1983). Foi 
classificada como hiperlordose lombar quando 0 valor da curvatura foi maior que $54^{\circ}$, e quando o valor da curvatura foi menor $22^{\circ}$ foi classificada como retificação lombar. Ainda, para análise das alterações da coluna vertebral, realizou-se uma classificação geral das curvaturas da coluna vertebral: com alteração na coluna vertebral (presença de alteração postural na torácica, lombar ou ambas), sem alteração da coluna vertebral (ausência de alteração postural na coluna vertebral na região torácica e lombar).

\section{Tratamento Estatístico}

Para o tratamento estatístico, através de procedimentos de estatística descritiva e inferencial, foi realizado no Software SPSS 18.0. As variáveis categóricas (1) alteração das curvaturas da coluna vertebral (com e sem alteração); (2) dor (com e sem dor) e (3) funcionalidade (com limitação e sem limitação) foram descritas utilizando-se tabelas de freqüência. A variável contínua qualidade de vida foi descrita por meio de média e desvio padrão.

Para verificar a associação entre alterações da coluna vertebral e dor, e alterações da coluna vertebral e funcionalidade utilizou-se o teste quiquadrado. O nível de significância adotado para este estudo foi de 0,05 .

\section{Resultados}

Participaram deste estudo 42 estudantes, sendo 8 (19\%) estudantes do sexo masculino e $34(81 \%)$ do sexo feminino. A Tabela 1 apresenta a caracterização da amostra segundo os valores médios de idade, massa corporal, estatura, IMC e RCQ.

Tabela 1. Caracterização da amostra (idade, estatura, massa corporal, Índice de Massa Corporal - IMC; Relação Cintura Quadril - RCQ).

\begin{tabular}{cc}
\hline Caracterização da Amostra & Média \pm Desvio padrão \\
\hline Idade (anos) & $20,3 \pm 3,4$ \\
Massa corporal $(\mathrm{Kg})$ & $61,5 \pm 9,9$ \\
Estatura $(\mathrm{m})$ & $1,6 \pm 0,1$ \\
$\mathrm{IMC}\left(\mathrm{Kg} / \mathrm{m}^{2}\right)$ & $21,9 \pm 2,2$ \\
$\mathrm{RCQ}$ & $0,7 \pm 0,1$ \\
\hline
\end{tabular}

A Tabela 2 apresenta a prevalência de dor nas costas, de alterações da coluna vertebral como um todo e de funcionalidade.

Tabela 2. Prevalência das alterações nas curvaturas da coluna vertebral, de dor nas costas e de funcionalidade.

\begin{tabular}{clcc}
\hline & Variáveis & Freqüência & Porcentagem (\%) \\
\hline Coluna Vertebral & Com alteração & 16 & 38,1 \\
$(\mathrm{n}=42)$ & Sem alteração & 26 & 61,9 \\
\hline Dor nas costas & Com dor & 29 & 69 \\
$(\mathrm{n}=42)$ & Sem dor & 13 & 31 \\
\hline Funcionalidade $^{*}(\mathrm{n}=29)$ & Com limitação & 1 & 3,4 \\
& Sem limitação & 28 & 96,6 \\
\hline
\end{tabular}

${ }^{*}$ Avaliada apenas nos estudantes que referiram sentir dor nas costas

Os resultados referentes à intensidade da dor nas costas demonstraram que 44,8\% $(n=13)$ referiram sentir dor fraca, $37,9 \% \quad(n=11)$ dor moderada, $13,8 \%(n=4)$ dor forte, $3,4 \%(n=1)$ dor muito forte.

Os resultados do teste de associação demonstraram que a dor nas costas não está associada significativamente $(p=0,618)$ com as alterações nas curvaturas da coluna vertebral. Do mesmo modo, verificou-se que a funcionalidade não está associada significativamente $(p=0,389)$ com as alterações nas curvaturas da coluna vertebral.

Os resultados referentes à avaliação das curvaturas da coluna vertebral, separadamente para a região torácica e região lombar, mensuradas com 0 arcômetro, estão apresentadas na Tabela 3. 
Tabela 3. Prevalência de alterações da coluna vertebral separada por região torácica e lombar.

\begin{tabular}{cccc}
\hline & Prevalências & Freqüência & Porcentagem (\%) \\
\hline \multirow{2}{*}{$\begin{array}{c}\text { Coluna Torácica } \\
(n=42)\end{array}$} & Retificada & 1 & 2,4 \\
& Normal & 35 & 83,3 \\
& Hipercifose & 6 & 14,3 \\
\hline \multirow{2}{*}{$\begin{array}{c}\text { Coluna Lombar } \\
(n=41)\end{array}$} & Retificada & 2 & 4,8 \\
& Normal & 29 & 69,0 \\
\end{tabular}

Os resultados referentes à avaliação qualidade de vida estão descritos na Tabela 4.

Tabela 4. Resultados do questionário SF-36 para cada um dos domínios: capacidade funcional, aspectos físicos, dor, estado geral de saúde, vitalidade, aspectos sociais, aspectos emocionais e saúde mental.

\begin{tabular}{lccc}
\multicolumn{1}{c}{ Domínios do SF36 } & mín & máx & Média \pm dp \\
\hline Capacidade Funcional & 70 & 100 & $90,4 \pm 8$ \\
Aspectos Físicos & 0 & 100 & $75,6 \pm 31,8$ \\
Dor & 20 & 54 & $36,7 \pm 9$ \\
Estado Geral de Saúde & 32 & 95 & $59,5 \pm 14,5$ \\
Vitalidade & 10 & 75 & $47,6 \pm 17,8$ \\
Aspectos Sociais & 0 & 100 & $67,5 \pm 27,6$ \\
Aspectos Emocionais & 0 & 100 & $61,6 \pm 40,9$ \\
Saúde Mental & 8 & 92 & $62,1 \pm 20$ \\
\hline
\end{tabular}

\section{Discussão}

Os resultados encontrados no presente estudo demonstraram uma prevalência de alterações na coluna vertebral de $38,1 \%$ e prevalência de dor nas costas de $69 \%$, sendo que destes estudantes com dor nas costas, apenas 3,4\% apresentaram limitação funcional. Martelli e Traebert (2006) avaliaram escolares por meio do posturógrafo e fio de prumo e encontraram uma prevalência de alterações posturais de $28,2 \%$, porém estes autores também avaliaram as alterações laterais, no plano frontal, além da avaliação no plano sagital. As duas alterações mais prevalentes foram a hiperlordose lombar $(20,3 \%)$ e hipercifose torácica (11\%). Estes resultados estão próximos aos resultados do presente estudo, em que a prevalência da hiperlordose lombar foi de $23,8 \%$ e de hipecifose torácica foi de $14,3 \%$.

Destsch et al. (2007) avaliaram as alterações laterais e ântero-posteriores de jovens do sexo feminino utilizando um posturógrafo e encontraram alta prevalência de alterações posturais. Os resultados do presente estudo demostraram uma prevalência de alterações da coluna vertebral de $38,1 \%$, sendo assim, um resultado que traz preocupação, pois a maioria também apresenta histórico de dor nas costas e isso poderia estar alterando a funcionalidade da coluna vertebral e a qualidade de vida dos universitários.

Karahan e Bayraktar (2004) avaliaram a postura corporal nas atividades diárias relacionadas ao trabalho e a existência de dor lombar de 56 enfermeiras, na Turquia. Os resultados mostraram que $87,5 \%$ das enfermeiras sofrem de dor lombar e que a maioria delas não utiliza o corpo na sua mecânica correta nas posturas laborais. Recentemente Najenson et al. (2010) demonstraram que $45,4 \%$ dos motoristas de ônibus avaliados (média de idade de 45 anos) apresentaram dor lombar no último ano, sendo que este resultado está de acordo com o presente estudo que apresentou dor nas costas em 69\% dos estudantes. Siqueira et al. (2008) realizou um estudo para verificar a ocorrência de lombalgia em fisioterapeutas e os resultados mostraram alto índice de alterações musculoesqueléticas na coluna lombar, uma vez que $78,56 \%$ dos fisioterapeutas relataram a presença de dor na região da coluna vertebral. Dezan et al. (2004), utilizando fotogrametria, realizaram um estudo com objetivo de analisar as curvaturas da coluna vertebral e a ocorrência de lombalgia em atletas, do sexo masculino e com idade média de 23,7 anos. Seus resultados mostraram uma prevalência de dor lombar de $58 \%$ e os autores concluíram que a dor lombar pode estar 
associada com alterações da coluna vertebral (aumento no ângulo torácico e lombar) e desequilíbrios musculares. Os achados do presente estudo não corroboram com estes resultados, uma vez que não foi encontrada relação significativa entre dor e alteração das curvaturas da coluna vertebral.

O estudo de $\underline{\text { Pinto }}$ et al. (2000) teve como objetivo verificar a relação entre 0 ângulo da lordose lombar e desempenho da musculatura abdominal em 50 alunos de fisioterapia. $O$ ângulo da lordose foi avaliado por radiografias enquanto que a força da musculatura abdominal foi avaliada por um esfigmomanômetro. Seus resultados demonstraram que não há correlação significativa entre estes parâmetros e que $36 \%$ dos estudantes apresentaram hiperlordose lombar, uma prevalência superior àquela encontrada no presente estudo que foi de 23,8\% (Tabela 3).

Falcão et al. (2007), utilizando fotogrametria, realizaram um estudo com estudantes universitários e funcionários para verificar a correlação de alterações posturais com dores musculoesqueléticas e os resultados demonstraram a não existência dessa associação, corroborando com os resultados do presente estudo, o qual também não encontrou associação entre alterações nas curvaturas da coluna, dor nas costas e funcionalidade, uma vez que a prevalência de alterações nas curvaturas da coluna foi de $38,1 \%$ e a prevalência da funcionalidade sem comprometimento foi alta. Não obstante, a dor nas costas, que apresentou alta prevalência, não causou incapacidade funcional, porém um acompanhamento longitudinal dos universitários seria interessante, já que quase $40 \%$ apresentam alteração da coluna vertebral.

Os resultados da avaliação da percepção da qualidade de vida dos estudantes de fisioterapia da Universidade Federal do Rio Grande do Sul, demonstraram que a maioria dos domínios do SF36 apresentou uma boa qualidade de vida, exceto o domínio da dor que apresentou um escore mais baixo. Esse resultado está de acordo com o resultado encontrado de alta prevalência de dor nas costas nos estudantes. O domínio da capacidade funcional apresentou um escore alto $e$ esse resultado também foi encontrado no questionário Roland-Morris que demonstrou alta prevalência de funcionalidade sem comprometimento. Tsukimoto et al. (2006) realizaram um estudo longitudinal em que os indivíduos (média da idade de 46,8 anos) com dor lombar participaram de uma Escola Postural, sendo avaliados por questionários de qualidade de vida e funcionalidade. Seus resultados mostraram que os indivíduos melhoraram significativamente nos domínios do SF-36 para capacidade funcional, aspectos físicos, dor, estado geral de saúde, vitalidade, bem como melhoraram seus escores do questionário Roland-Morris.

Finalizando, entende-se interessante comentar que o presente estudo utilizou 0 instrumento arcômetro para avaliar a coluna vertebral, pois é considerada uma ferramenta alternativa, de fácil aplicabilidade, baixo custo, fácil manuseio e que permite uma avaliação quantitativa e objetiva das curvaturas da coluna vertebral (CHAISE et al., 2011). Tradicionalmente, nos estudos de prevalência, a avaliação da coluna vertebral tem sido realizada através da observação da sua forma, ou seja, pela inspeção visual, seja por fotografias ou não, sendo o instrumento posturógrafo o mais comumente utilizado. Nesse sentido, o presente estudo diferencia-se quando apresenta a possibilidade da obtenção de medidas diretas das curvaturas. Não obstante, a opção por este instrumento apresenta uma importante limitação, que é a impossibilidade de avaliar a postura dos estudantes como um todo.

\section{Conclusão}

Os resultados demonstraram prevalência de alterações nas curvaturas da coluna vertebral de $38,1 \%(n=16)$, prevalência de dor nas costas de $69 \%$; $(n=29)$ e prevalência de comprometimento da funcionalidade de $3,4 \%$; $(n=1)$.

Os resultados não demonstraram associação significativa entre dor nas costas e as alterações nas curvaturas da coluna, e entre funcionalidade e as alterações nas curvaturas da coluna. Os resultados da avaliação da qualidade de vida mostraram que os escores de todos os domínios foram maiores que 45 , exceto o domínio "dor", que foi de 36,7 .

Entende-se interessante reavaliar esses estudantes com intuito de verificar a evolução do quadro apresentado, no que tange as alterações da coluna vertebral, a dor nas costas, a capacidade funcional e a qualidade de vida dos mesmos. 


\section{Referências}

BARAÚNA, M.A.; et al. Validade e confiabiliadade intra-indivíduo do cifolordômetro na avaliação da convexidade torácica. Revista Brasileira de Fisioterapia, São Carlos, v. 9, n.3, p. 319-325, 2005.

BERNHARDT, M.; BRIDWELL, K. Segmentar analysis of the sagital plane alignment of the normal thoracic and lumbar spines ond thoracolumbar junction. Spine, (Phila $\mathrm{Pa} 1976) \mathrm{v}$. 14, n. 7, p. 717-721, 1989. Disponível em: http://dx.doi.org/10.1097/00007632-198907000$\underline{00012}$.

CAILLIET, R. Síndrome da Dor Lombar. 5a edição. Porto Alegre: Artmed, 2001.

CANDOTTI, C.T.; GUIMARÃES, A.S. O emprego do método de relaxamento muscular de Leon Michaux no tratamento da dor lombar de atletas de ginástica rítmica desportiva. Revista Perfil, Porto ALegre, v. 2, n. 2, p. 19-27, 1998.

CANDOTTI, C.T.; NOLL, M.; CRUZ, M. Prevalência de dor lombar e os desequilíbrios musculares em manicures. Revista Arquivos em Movimento, Rio de Janeiro, v.6, n.1, p.125-40, 2010.

CARNEIRO, J.A.O.; SOUSA, L.M.S.; MUNARO, H.L.R. Predominância de desvios posturais em estudantes de Educação Física da Universidade Estadual do Sudoeste da Bahia. Revista Saúde Comunitária, v. 1, n. 2, p. 118-123, 2005.

CASTRO, P.C.G.; LOPES, J.A.F. Avaliação computadorizada por fotografia digital, como recurso de avaliação na reeducação postural global. Acta Fisiátrica, São Paulo, v. 10, n. 2, p. 83-88, 2003.

CHAISE, F.O.; et al. Validade, repetibilidade e reprodutibilidade de um instrumento não-invasivo para medição das curvaturas torácica e lombar da coluna vertebral no plano sagital. Revista

Brasileira de Fisioterapia, São Carlos, v.5, n.6, p.511-517, 2011. Disponível em: http://dx.doi.org/10.1590/S141335552011005000031.

CIENA, A.P.; et al. Influência da intensidade da dor sobre as respostas nas escalas unidimensionais de mensuração da dor em uma população de idosos e de adultos jovens.

Semina: Ciências Biológicas e da Saúde, Londrina, v. 29, n.2, p. 201-212, 2008.

CICONELLI, R.M.; et al. Tradução para a língua portuguesa e validação do questionário genérico de qualidade de vida SF-36 (Brasil SF-36).
Revista Brasileira Reumatologia, Campinas, v. 39, n. 3, p. 143-150, 1999.

D'OSUALDO, F.; SCHIERANO, S.; IANNIS, M. Validation of clinical measurement of kyphosis with a simple instrument, the arcometer. Spine, v. 22, p. 408-413, 1997.

DETSCH, C.; et al. Prevalência de alterações posturais em escolares do ensino médio em uma cidade no Sul do Brasil. Revista Panamericana de Salud Pública, Washington, v. 21, n. 4, p. 231-238, 2007. Disponível em: http://dx.doi.org/10.1590/S1020$\underline{49892007000300006 .}$.

DIAS, M.B.; et al. Desenvolvimento de um arcômetro para mensuração das curvaturas sagitais torácica e lombar da coluna vertebral. In: XII Congresso Ciências do Desporto e Educação Física dos Países de Língua Portuguesa, 2008, Porto Alegre. Caderno de Resumos do XII Congresso Ciências do Desporto e Educação Física dos Países de Língua Portuguesa, $p$. 303, 2008.

DIEËN, J.H; SELEN, L.P.J; CHOLEWICHI, J. Trunk muscle activation in low-back pain patients, an analysis of the literature. Journal of Electromyography and Kinesiology, New York, v. 13, p. 333-351, 2003. Disponível em: http://dx.doi.org/10.1016/S1050-6411(03)00041-5.

DEZAN, V.H.; SARRAF, T.A.; RODACKI, A.L.F. Alterações posturais, desequilíbrios musculares e lombalgias em atletas de luta olímpica. Revista Brasileira Ciência e Movimento, São Paulo, v. 12, n. 1, p. 35-38, 2004.

DOODY, M.M.; et al. Breast cancer mortality after diagnostic radiography. Spine, (Phila Pa 1976,) v. 25, p. 2052-2063, 2000.

FALCÃO, F.R.C.; MARINHO, A.P.S.; SÁ, K.N. Correlação dos desvios posturais com dores músculo-esqueléticas. Revista de Ciências Médicas e Biológicas, Salvador, v. 6, n. 1, p. 5462, 2007.

GAYA, A.; et al. Ciências do movimento humanoIntrodução à metodologia da pesquisa. Porto Alegre: Artmed, 2008.

HINMANN, M.R. Comparison of thoracic kyphosis and postural stiffness in younger and older women. The Spine Journal, v. 4, p. 413-417, 2004.

KARAHAN, A.; BAYRAKTAR, N. Determination of the usage of body mechanics in clinical settings and the occurrence of low back pain in nurses. International Journal of Nursing Studies, 
Oxford, v.41, p. 67-75, 2004. Disponível em: http://dx.doi:10.1016/S0020-7489(03)00083-X.

KENDALL, F.P.; McCREARY, E.K.; PROVANCE, P.G. Músculos provas e funções. $5^{a}$ edição. São Paulo: Manole. 2007.

KNOPLICH, J. Enfermidades da Coluna Vertebral: uma visão clinica e fisioterápica. $3^{\underline{a}}$ ed. São Paulo: Robe editorial, 2003.

LEROUX, M.A.; et al. A Noninvasive Anthropometric Technique for Measuring Kyphosis and Lordosis. Spine, v. 25, n. 13, p. 1689-1694, 2000.

MARTELLI, R.C.; TRAEBERT, J. Estudo descritivo das alterações posturais da coluna vertebral em escolares de 10 a 16 anos de idade. Tangará-SC, 2004. Revista Brasileira de

Epidemiologia, São Paulo, v. 9, n. 1, p. 87-93, 2006. Disponível em:

http://dx.doi.org/10.1590/S1415790X2006000100011..

NAJENSON, D.; et al. Low Back Pain among Professional Bus Drivers: Ergonomics and Occupational - Psychosocial Risk Factors. Israel Medical Association Journal, Israel, v. 12, p. 2631, 2010.

NUSBAUM, L.; et al. Translation, adaptation and validation of the Roland-Morris questionnaire -Brazil Roland-Morris. Brazilian Journal of Medical and Biological Research, Ribeirão Preto, v. 34, p. 203-210, 2001.

OCARINO, J.M.; et al. Correlação entre um questionário de desempenho funcional e testes de capacidade física em pacientes com lombalgia.

Revista Brasileira de Fisioterapia, São Carlos, v.13, n.4, 2009. Disponível em: http://dx.doi.org/10.1590/S141335552009005000046.

PINTO, P.R.; et al. Relação entre lordose lombar e desempenho da musculatura abdominal em alunos de fisioterapia. Acta Fisiátrica, São Paulo, v. 7, n. 3, p. 95-98, 2000.

PROPST-PROCTOR, S.L.; BLECK, E.E. Radiographic determination of lordosis and kiphosis in normal and scoliotic children. Journal of Pediatric Orthopedics, New York, v. 3, p. 344346, 1983.

SIQUEIRA, G.R., CAHÚ, F.G.M., VIEIRA, R.A.G. Ocorrência de lombalgia em fisioterapeutas da cidade de Recife, Pernambuco. Revista

Brasileira de Fisioterapia, São Carlos, v. 2, n. 3, p. 222-227, 2008. Disponível em: http://dx.doi.org/10.1590/S141335552008000300010 .
TRIBASTONE, F. Tratado de exercícios corretivos aplicados à reeducação motora postural. São Paulo: Manole, 2001.

TSUKIMOTO, G.R.; et al. Avaliação longitudinal da Escola de Postura para dor lombar crônica através da aplicação dos questionários Roland Morris e Short Form Health Survey (SF-36). Acta Fisiátrica, São Paulo, v. 13, n. 2, p. 63-69, 2006.

VIGATTO, H, R.N.; COSTA, N.M.; CORREA, H.R. Development of a Brazilian Portuguese Version of the Oswestry Disability Index. Cross-Cultural Adaptation, Reability and Validity. Spine, v. 32, n. 4, p. 481-486, 2007.

\section{Endereço:}

Cláudia Tarragô Candotti

UFRGS - Escola de Educação Física (Sala 218)

Rua Felizardo 750 Bairro: Jardim Botânico

Porto Alegre RS Brasil

90690-200

Telefone: (51) 3308.5861

e-mail: claudia.candotti@ufrgs.br

Recebido em: 23 de outubro de 2011.

Aceito em: 01 de abril de 2013.

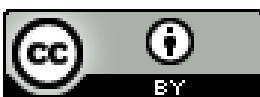

Motriz. Revista de Educação Física. UNESP, Rio Claro, SP, Brasil - elSSN: 1980-6574 - está licenciada sob Creative Commons - Atribuição 3.0 